\title{
The incidence of prostate cancer and urothelial cancer in the prostate in cystoprostatectomy specimens in a tertiary care Canadian centre
}

\author{
Sri Sivalingam, MD, MSC, FRCSC; Darrel Drachenberg, MD, FRCSC
}

Department of Urology, University of Manitoba, Winnipeg, MB

See related article on page 39 .

Cite as: Can Urol Assoc J 2013;7:35-8. http://dx.doi.org/10.5489/cuai.212

\section{Abstract}

Introduction: Radical cystoprostatectomy remains the gold standard treatment for muscle invasive bladder cancer. However, given the treatment related complications of compromised potency and continence with this procedure, prostate/sexuality sparing cystectomy in orthotopic neobladder candidates has emerged in an effort to minimize these quality of life concerns. Recent evidence suggests only a marginal functional benefit from these technical refinements. We sought to determine the incidence of occult prostate cancer and urothelial cancer of the prostate in cystoprostatectomy specimens conducted for muscle invasive bladder cancer.

Methods: We retrospectively reviewed 83 male patients who underwent radical cystoprostatectomy for muscle invasive bladder cancer between April 2004 and March 2007. The median age of our study group was 71 years. Pathologic findings of prostate/urothelial cancer in the prostate were identified. Clinically significant prostate cancer was defined as Gleason score $>6$, tumour volume $>0.5 \mathrm{cc}$, extracapsular extension or perineural invasion.

Results: Our review yielded a $30 \%( \pm 10 \%, 0.95 \mathrm{Cl})$ rate of prostate cancer, with $19 \%( \pm 8.5 \%, 0.95 \mathrm{Cl})$ of total specimens being positive for clinically significant prostate cancer. Urothelial cancer in the prostate was identified in $16 \%( \pm 8.5 \%, 0.95 \mathrm{Cl})$ of patients, with an overlap with prostate cancer in 2 patients. The overall rate of an underlying cancer within the prostate of our cystoprostatectomy specimens was about $46 \%( \pm 10.7 \%, 0.95 \mathrm{Cl})$.

Conclusion: These findings suggest that the oncological risk of leaving behind residual cancer may not justify the practice of prostatesparing cystectomies.

\section{Introduction}

The gold standard treatment for localized high grade muscleinvasive carcinoma of the bladder is radical cystectomy and bilateral pelvic lymph node dissection with concomitant removal of the prostate, seminal vesicles and vas deferens. ${ }^{1}$ Although there is an increasing trend towards early cystectomy for high-grade, non-muscle invasive bladder cancer, ${ }^{2}$ this procedure carries significant morbidities even with advances in "nerve-sparing" cystoprostatectomy. Morbidities include urinary incontinence and erectile dysfunction; these quality of life issues can curtail the patient's decision to choose extirpative surgery as primary treatment and thus delay definitive therapy. ${ }^{3}$ In view of this, ongoing modifications of the standard technique to improve the treatment related morbidities has led to prostate-sparing cystectomy. ${ }^{4}$ Prostatesparing cystectomy is defined as partial or complete sparing of the prostate, seminal vesicles or vas deferens during cystectomy with the purpose of minimizing dissection near the neurovascular bundles and external urinary sphincter. ${ }^{5}$

The use of prostate-sparing cystectomy raises concerns about the oncologic efficacy of urothelial cancer treatment and the potential impact of residual occult prostatic malignancy on long-term cancer recurrence and survival. To lower the risk of future malignancy following prostatesparing cystectomy, strict screening protocols are used to rule out adenocarcinoma and urothelial carcinoma of the prostate before surgery. ${ }^{6}$ These measures do not preclude future development of malignancies, and definitive treatment can be more challenging following cystectomy given the loss of tissue planes and peri-prostatic inflammatory and fibrotic changes that occur with requisite urine leakage following the original prostate-sparing cystectomy neobladder surgery. Additionally, the anticipated benefit in erectile function and urinary continence has not been ascertained. ${ }^{7}$

At our Canadian tertiary care referral centre, prostatesparing cystectomy is not generally offered, as radical cystoprostatectomy (RCP), including nerve-sparing RCP for potent individuals, remains the standard procedure for definitive surgical management of muscle invasive bladder cancer. The objective of this study was to determine the incidence of occult clinically significant adenocarcinoma and urothelial cancer of the prostate in our cystoprostatectomy series. 
This information could then serve as an indicator of what proportion of our patient population might be disadvantaged by prostate-sparing surgery.

\section{Methods}

We retrospectively reviewed 83 consecutive male patients who underwent RCP for muscle invasive bladder cancer at the University of Manitoba between April 2004 and March 2007. Institutional research ethics board approval was obtained prior to data collection. Inclusion criteria were males undergoing RCP for bladder cancer, as standard of care treatment; male patients who underwent RCP not related to bladder cancer and female patients were excluded. None of the patients had known or clinical suspicion of prostate cancer at the time of RCP. Of the total 104 cases, 83 met the inclusion criteria; median patient age was 71 years (range: 43-85). Surgical pathology reports were reviewed to stratify prostate pathology as benign, prostate adenocarcinoma or urothelial cancer of the prostate. Clinically significant prostate cancer was defined as tumour diameter $>1.0 \mathrm{~cm}$ (as an approximate surrogate for volume $>0.5 \mathrm{cc}$ [i.e., a $1-\mathrm{cm}$ diameter would have a maximum volume of $0.5 \mathrm{cc}$ if spherical, based on $4 / 3 \Pi \mathrm{r}^{3}$, with elliptoid tumours having lesser volumes]), Gleason score $\geq 7$ or extracapsular/ perineural/seminal vesicle invasion. ${ }^{8}$ Prostatic intraepithelial neoplasia was also identified, but in this population was not considered clinically significant. Statistical analysis was based on $95 \%$ confidence limits for proportion based on Wilson's method.

\section{Pathologic analysis}

The prostatic soft tissue margins and seminal-vesical margins were painted with black ink; the prostate was sectioned in the transverse plane at 5-mm intervals from apex to base. Sectioning of the prostate was based on a semi-standardized protocol. If no gross tumour was identified, prostate sections included:

1. Either an en-face prostatic urethral margin or a complete radial submission of the prostatic apex, including the entire urethral margin. In the former case, additional random sections of apex were performed.

2. Two random sections of each prostatic quadrant.

3. One random section from each prostatic base (each side).

4. One random section from each seminal vesicle.

If the prostate tumour was identified grossly, a more extensive standardized partial grossing protocol was used involving complete gross tumour submission and 4 sections per quadrant. If grossly unsuspected prostate cancer was identified on microscopic examination, further gross submission was at the discretion of the sign-out pathologist.
Histologic sectioning was performed as per institutional protocols. $^{9}$

\section{Results}

Of the 83 cases reviewed, 25 patients had prostate adenocarcinoma, corresponding to a $30 \%( \pm 10 \%, 0.95 \mathrm{Cl})$ rate of occult prostate cancer. Twelve patients $(15 \% \pm 8 \%, 0.95 \mathrm{Cl})$ had clinically significant prostate cancer: 10 had Gleason score $\geq 7$ and 2 had one or more of the other predefined clinically significant factors described previously (Table 1 , Table 2). There were 8 specimens with high-grade prostatic intraepithelial neoplasia. Of all prostate adenocarcinoma detected, $48 \%( \pm 20 \%, 0.95 \mathrm{Cl})$ were classified as clinically significant. Urothelial cancer in the prostate was identified in $13(16 \% \pm 9 \%, 0.95 \mathrm{Cl})$ patients, with an overlap with prostate cancer in 2 of these patients.

Pathological analysis of the 13 cases with transitional cell carcinoma (TCC) revealed prostatic stromal involvement in 6 , contiguous TCC in 3, and non-contiguous prostatic urethral TCC in 4 (Table 3). The overall rate of an underlying cancer (i.e., prostate adenocarcinoma or urothelial cancer of the prostate) within the prostate of our cystoprostatectomy specimens was about $46 \%( \pm 10.7 \%, 0.95 \mathrm{Cl})$. The combined rate of clinically significant prostate adenocarcinoma and urothelial cancer of the prostate was $27 \%$. Follow-up data were not included in this study.

\section{Discussion}

The functional losses and diminished quality of life resulting from RCP has led to many technical refinements over the years, such as nerve-sparing and prostate-sparing cystectomy. Implicit in such modifications is a deviation from oncologic principles of removing at-risk tissue/organs enblock with the diseased organ; however, this tissue-sparing approach is attractive for the potential functional benefits..$^{4,10}$ Common morbidities include urinary incontinence and sexual dysfunction, even in the hands of experienced surgeons. Prostate-sparing cystectomy is purported to offer a higher incidence of erectile nerve preservation and sphincteric preservation with improvement in potency and continence,

\begin{tabular}{lcc}
\hline \multicolumn{3}{l}{ Table 1. Adenocarcinoma/urothelial cancer in the prostate } \\
\hline & N & $\%( \pm 95 \% \mathbf{C l})$ \\
\hline Total prostate cancer & 25 & $30( \pm 10)$ \\
Clinically significant prostate cancer* & 12 & $15( \pm 8)$ \\
High grade PIN & 8 & $10( \pm 6)$ \\
Extracapsular extension & 4 & $5( \pm 5)$ \\
Perineural invasion & 7 & $8( \pm 6)$ \\
Urothelial cancer within prostate & 13 & $16( \pm 9)$ \\
\hline *Tumour diameter $>1$ cm, Gleason Score $>6$, extracapsular/perineural/seminal vesicle \\
invasion. PIN: prostatic intraepithelial neoplasia.
\end{tabular}




\begin{tabular}{lc}
\hline $\begin{array}{l}\text { Table 2. Gleason score } \\
\text { distribution }\end{array}$ \\
\hline Grade & N \\
\hline 4 & 1 \\
5 & 2 \\
6 & 12 \\
7 & 8 \\
8 & 0 \\
9 & 2 \\
10 & 0 \\
HPIN & 8 \\
\hline HPIN: High-grade prostatic & \\
intraepithelial neoplasia & \\
\hline
\end{tabular}

especially for younger males. ${ }^{11}$ The first large series describing the outcomes of prostate-sparing cystectomy boasted $97 \%$ daytime continence rates at 1 year and $82 \%$ preservation of potency..$^{12}$ However, in contemporary studies, good daytime continence has been achieved after orthotopic bladder substitution in many series, but with varying degrees of nocturnal enuresis in $20 \%$ to $25 \%$ of patients. The larger benefit may be seen in the preservation of potency, with rates over $80 \% .5,13,14$

A caveat of prostate-sparing cystectomy is that patients need to be highly selected based on pre-existing function and screened to rule out underlying malignancy within the prostate. Qualifying patients must be motivated for preserving pre-existing sexual function; patients with a tumour at the bladder neck or prostatic urethra must be excluded. ${ }^{15}$ Any patient who chooses prostate-sparing cystectomy will necessarily have to be screened with one or more invasive procedures prior to surgery, including transurethral resection of bladder neck/prostatic urethra to rule out tumour within these structures and transrectal ultrasound biopsy of the prostate along with prostate-specific antigen and digital rectal exam to rule out prostate adenocarcinoma. ${ }^{16}$ Preoperative screening should be interpreted cautiously, as transurethral biopsy is not a reliable indicator of prostatic involvement in bladder cancer. ${ }^{17}$ Furthermore, understanding that this procedure is most often offered to young patients, patients undergoing prostate-sparing procedures will need years of ongoing screening for prostate cancer, as well as surveillance for local recurrence of urothelial cancer. Unfortunately, there are no validated screening protocols for risk stratification of occult prostatic malignancy in cystectomy patients - this raises further concern in standardizing the prostate-sparing cystectomy approach. ${ }^{5}$ In identifying unsuitable candidates for prostate-sparing cystectomy, oncological factors, such as distance of the bladder tumour from the bladder neck or presence of solitary T2-T3 bladder tumours at the trigone or bladder neck, are predictive of invasive prostatic involvement. ${ }^{18-20}$ These data can help identify patients who will not be suitable for prostate-sparing cystectomy.
Before we can adopt prostate-sparing cystectomies as a routine option for muscle invasive bladder cancer, the potential risk of residual occult cancer in the prostate must considered. Accordingly, we assessed the rates of cancer within the prostate (prostate adenocarcinoma and TCC of the prostate) in our series of RCPs. We found clinically significant prostate adenocarincoma in $15 \%$ of our samples; when combined with the risk of occult urothelial cancer, the overall risk of significant disease is $27 \%$. This rate is within observed rates; however, rates vary widely from less than $10 \%$ and up to $60 \% .{ }^{16}$ The variation in the observed rates may be explained by factors, such as tissue sampling protocols (i.e., slice thickness), variability in pathologic examination protocols, definition of clinical significance, and the degree of inclusiveness of the prostatic tissue. ${ }^{21}$ The implication of this is that ongoing screening is mandatory, and it remains that if clinically significant prostate cancer exists or develops, prior prostate-sparing cystectomy makes further surgery difficult. Additionally, the bowel segment of a neobladder may hinder the ability to deliver curative radiotherapy, leaving fewer treatment options. Conversely, in patients who undergo RCP, removal of the prostate obviates surveillance for prostate cancer. ${ }^{22}$

No randomized controlled trials have been conducted to date to compare prostate-sparing cystectomy versus RCP regarding oncologic and functional outcomes. Hautmann and colleagues reviewed organ preserving cystectomies in the past 15 years; their results showed higher rates of distant failure when compared to standard or nerve-sparing cystectomies, and the authors concluded such an approach was a step backwards in oncologic efficacy. ${ }^{7}$ Of note, in their review involving 13 centres worldwide, there was no benefit in preserving continence, although a significant improvement in potency rates was shown. ${ }^{7}$ Saad and colleagues reported that the potential oncologic risks of prostate-sparing cystectomy outweigh any small and possible functional benefits. ${ }^{13}$ Contrary to this, other authors contend that prostate-sparing cystectomy is oncologically safe if offered to highly selected patients with disease-specific survival rates; in these patients, local recurrence rates are comparable to the standard approach, ${ }^{4}$ and potency rates may even be better. ${ }^{5}$ Finally, in the prospective series by Rozet and colleagues, oncologic outcomes on prostate-sparing cystectomy patients were comparable to the largest published series on cystoprostatectomies, suggesting that for carefully selected patients, this may be a viable additional option for treatment. ${ }^{23}$ Despite currently existing data, further follow-up with long-term data is needed to determine the oncologic efficacy of prostate-sparing cystectomy.

Our results are consistent with the current literature, indicating a substantial rate of clinically significant occult prostatic adenocarincoma or prostatic involvement of urothelial cancer. Until further evidence emerges to confirm 
the oncologic safety and functional benefits of performing prostate-sparing cystectomies, prostate-sparing cystectomy cannot be considered standard of care and should only be carefully considered in highly selected, motivated patients. In centres that offer prostate-sparing cystectomy, patients, in addition to be properly screened for prostatic malignancies in the residual tissue, must be carefully counselled in regards to their expectations of functional outcomes and understand the risks. It should also be noted that these results only look at the risk of concurrent malignancies that could compromise oncologic efficacy at the time of RCP. Thus, it underestimates overall risk to these patients given that it is currently impossible to estimate future oncologic risk for developing prostatic malignancy. Given the natural history of prostate cancer, it may be the same young patients for whom we wish to minimize impact on quality of life by prostate-sparing cystectomy that stand the most to lose by developing prostate cancer years after surgery and later in life.

\section{Conclusions}

Our findings suggest that the oncological risk of leaving behind residual cancer may not justify the practice of prostate-sparing cystectomy as standard of care and that this procedure should be reserved for highly motivated and highly selected patients. Randomized controlled clinical trials remain the only way to evaluate the oncologic safety and quality of life endpoints of prostate-sparing cystectomy versus RCP.

Competing interests: None declared.

This paper has been peer-reviewed.

Acknowledgements: We would like to thank Dr. Robert Wightman for help with pathologic considerations and Dr. Karen Psooy for her contribution with draft revisions.

\section{References}

1. Stein JP, Skinner DG. Radical cystectomy for invasive bladder cancer: long-term results of a standard procedure. World J Urol 2006;24:296-304. http://dx.doi.org/10.1007/s00345-006-0061-7

2. Bochner BH. Optimal timing of radical cystectomy for patients with T1 bladder cancer. Urol Oncol 2009;27:329-31. http://dx.doi.org/10.1016/i.urolonc.2008.10.022

3. Fahmy NM, Mahmud S, Aprikian AG. Delay in the surgical treatment of bladder cancer and survival: systematic review of the literature. Eur Urol 2006;50:1176-82. http://dx.doi.org/10.1016/i eururo.2006.05.046
4. de Vries RR, Nieuwenhuïzen JA, van Tinteren H, et al. and Center UWGotACC. Prostate-sparing cystectomy: long-term oncological results. BJU Int 2009;104:1239-43. http://dx.doi.org/10.1111/i.1464410X.2009.08615.x

5. Kefer JC, Campbell SC. Current status of prostate-sparing cystectomy. Urol Oncol 2008;26:486-93. http://dx.doi.org/10.1016/i.urolonc.2008.03.018

6. Horenblas $S$, Meinhardt $W$, lizerman $W$, et al. Sexuality preserving cystectomy and neobladder: initial results. J Urol 2001;166:837-40. http://dx.doi.org/10.1016/S0022-5347(05)65847-8

7. Hautmann RE, Stein JP. Neobladder with prostatic capsule and seminal-sparing cystectomy for bladder cancer: a step in the wrong direction. Urol Clin North Am 2005;32:177-85. http://dx.doi.org/10.1016/i. ucl.2005.02.001

8. Epstein Jl, Chan DW, Sokoll U, et al. Nonpalpable stage Tlc prostate cancer: prediction of insignificant disease using free/total prostate specific antigen levels and needle biopsy findings. J Urol 1998;160:240711. http://dx.doi.org/10.1016/S0022-5347(01)62200-6

9. Wightman R. Pathological protocols for radical cystoprostatectomy specimens. University of Manitoba Department of Pathology Institutional Protocol 2009.

10. Nieuwenhuïzen JA, Meinhardt W, Horenblas $S$. Clinical outcomes after sexuality preserving cystectomy and neobladder (prostate sparing cystectomy) in 44 patients. J Urol 2008; 179:S35-8. http://dx.doi. org/10.1016/i.juro.2008.03.135

11. Morris DS, Weizer AZ, Ye $Z$, et al. Understanding bladder cancer death: tumour biology versus physician practice. Cancer 2009:115:1011-20. http://dx.doi.org/10.1002/cncr.24136

12. Vallancien $G$, Abou El Fettouh $H$, Cathelineau $X$, et al. Cystectomy with prostate sparing for bladder cancer in 100 patients: 10-year experience. J Urol 2002;168:2413-7. http://dx.doi.org/10.1016/ S0022-5347(05)64157-2

13. Saad M, Abdel-Rahim M, Abol-Enein H, et al. Concomitant pathology in the prostate in cystoprostatectomy specimens: a prospective study and review. BJU Int 2008;102:1544-50. http://dx.doi.org/10.1111/ j.1464-410X.2008.07831.x

14. Hautmann RE, de Petriconi R, Gottfried HW, et al. The ileal neobladder: complications and functional results in 363 patients after 11 years of followup. J Urol 1999;161:422-7; discussion 427-8. http:// dx.doi.org/10.1016/50022-5347(01)61909-8

15. Kefer JC, Voelzke BB, Flanigan RC, et al. Risk assessment for occult malignancy in the prostate before radical cystectomy. Urology 2005;66:1251-5. http://dx.doi.org/10.1016/i.urology.2005.07.006

16. Autorino $R$, Di Lorenzo $G$, Damiano $R$, et al. Pathology of the prostate in radical cystectomy specimens: a critical review. Surg Oncol 2009;18:73-84. http://dx.doi.org/10.1016/i.suronc.2008.07.006

17. Donat SM, Wei DC, McGuire MS, et al. The efficacy of transurethral biopsy for predicting the long-erm clinical impact of prostatic invasive bladder cancer. J Urol 2001;165:1580-4. http://dx.doi.org/10.1016/ S0022-5347(05)66352-5

18. Arce J, Gaya JM, Huguet J, et al. Can we identify those patients who will benefit from prostate-sparing surgery? Predictive factors for invasive prostatic involvement by transitional cell carcinoma. Can I Urol 2011:18:5529-36

19. Tabibi A, Simforoosh $N$, Parvin $M$, et al. Predictive factors for prostatic involvement by transitional cell carcinoma of the bladder. Urol J 2011;8:43-7.

20. Richards KA, Parks GE, Badlani GH, et al. Developing selection criteria for prostate-sparing cystectomy: a review of cystoprostatectomy specimens. Urology 2010;75:1116-20. http://dx.doi.org/10.1016/i. urology.2009.09.081

21. Mazzucchelli R, Barbisan F, Scarpelli M, et al. Is incidentally detected prostate cancer in patients undergoing radical cystoprostatectomy clinically significant? Am I Clin Pathol 2009;131:279-83. http://dx.doi. org/10.1309/AJCP40CYZBAN9TJU

22. Bivalacqua TJ, Loeb S, Pierorazio PM, et al. Is prostate-specific antigen surveillance necessary in men with benign prostate pathology following radical cystoprostatectomy for bladder cancer? Urol Int 2010;85:4669. http://dx.doi.org/10.1159/000321693

23. Rozet $F$, Lesur $G$, Cathelineau $X$, et al. Oncological evaluation of prostate sparing cystectomy: the Montsouris long-term results. J Urol 2008;179:2170-4; discussion 2174-5. http://dx.doi.org/10.1016/i. juro.2008.01.112

Correspondence: Dr. Darrel Drachenberg, Director of Research, Section of Urology and Manitoba Prostate Centre, Chair of Genitourinary Oncology Disease site group, Cancer Care Manitoba, Department of Surgery, University of Manitoba, Winnipeg, MB; DDRACHENBERG@sbgh.mb.ca 